\title{
Smartphone-based Identification of Critical Levels of Glycated Hemoglobin A1c using Transdermal Optical Imaging
}

\author{
HUDDA SALIH ${ }^{1 ’ 2}$, SI JIA WU ${ }^{3}$, EVGUENI KABAKOV ${ }^{3}, K^{\prime}$ KNG LEE ${ }^{3}$, AND WEIHONG ZHOU ${ }^{1}$ \\ 1 Department of Health Management Centre, Nanjing Drum Tower Hospital, China \\ 2 Department of Biological Sciences, University of Toronto Scarborough, Canada \\ ${ }^{3}$ Applied Psychology and Human Development, University of Toronto, Canada
}

\section{Abstract}

A current health concern is the constraints of blood glucose monitoring techniques in the face of the ever-expanding predominance of diabetes. Electronic medical devices can potentially overcome these limitations and prevent the development of diabetes-related complications. This study investigated whether advanced machine learning methods and Anura, a smartphone-based transdermal optical imaging technology (TOI) that assesses health markers, can be a viable solution for diabetes management. To examine the validity of TOI and a novel machine algorithm for diabetes prediction, we compared the diabetes classification from TOl's obtained glycated hemoglobin A1c (HbA1c) concentrations against data obtained from FDA-approved blood immunoassays. The data set was obtained from 513 participants recruited during their annual physical examination at the Health Management Centre of the Affiliated Hospital of Nanjing University Medical School, China. We used a kitchen sink random forest machine algorithm for diabetes prediction. To validate the model, pristine testing was done on 400 pristine participants pseudo-randomly selected during 20 trials of training and testing. The confusion matrix found TOI to have a classification accuracy of $66 \%$, and the Receiver operating characteristic (ROC) curve of the Random Forest (RF) classifier found TOI to have a ROC Area Under the Curve (AUC) of .69. The present study provides evidence for the potential use of the TOl technology, Anura, for contactless, non-invasive, and inexpensive assessments of diabetes.

Keywords: transdermal optical imaging, diabetes, glycated hemoglobin A1C

\section{Introduction}

The prevalence of diabetes is rising at an alarming rate, nearing the proportions of a global epidemic. Currently, the number of adults with diabetes is 463 million and is expected to rise to 700 million by 2045
(Saeedi et al., 2019). In diabetes, the absolute deficiency of insulin and/or insulin resistance does not allow glucose to sufficiently leave the bloodstream to enter cells of the body (University of Leicester, 2016). This results in an 
increased blood glucose level. High blood glucose, or hyperglycemia, is a major concern of those affected with diabetes. A high glycemic level causes behavioural changes in arterial myocytes, leading them to contract more than they would at normal blood glucose levels (University of Leicester, 2016). This can cause high blood pressure and reduce the supply of blood to vital organs (University of Leicester, 2016).

Recognizing the signs of high and low blood glucose is crucial to one's health. Every six seconds, someone is estimated to die from diabetes-related complications, and in 2013, diabetes caused approximately 5.1 million deaths (Bird et al., 2015). Although monitoring and controlling glucose levels are essential to prevent the development of diabetesrelated complications and death, the current assessment methods have numerous limitations. We still face difficulties in reliably and conveniently monitoring glucose. The present research aimed to address these difficulties with the use of transdermal optical imaging (TOI), a novel smartphone-based technology for contactless assessment of physiological health and well-being.

Two major methods have been used to monitor glucose levels. The first is the glucose test (Sherwani et al., 2016). This approach involves the extraction of patients' blood in order to measure their blood glucose levels (Solnica \& Naskalski, 2007). While this method is an integral part of diabetes care, it presents numerous limitations (Ong et al., 2014). Glucose tests provide measures in real-time, so they are affected by multiple factors such as illness and food ingestion (Qian \& Scheer, 2016). In addition, glucose metabolism is under circadian control; thus, the measure is also influenced by the large daily fluctuations that result from blood glucose regulation (Qian \& Scheer, 2016).

The second method, the glycated hemoglobin $\mathrm{A} 1 \mathrm{c} \quad(\mathrm{HbA} 1 \mathrm{c})$ test, is considered the gold standard assessment for diabetes management. The test measures the amount of glucose attached to the HbA1c protein, consequently giving an average blood glucose level over the three-month life span of the protein (Sherwani et al., 2016). While the HbA1c test overcomes certain limitations of the glucose test, it does require blood samples collected by physicians to undergo laboratory assessment and places a constant burden on the household budget (Sherwani et al., 2016). For appropriate diabetes management, diabetics are recommended to take the HbA1c test every 3 months ( $\$ 44$ per test) and self-monitor their blood glucose ( $\$ 860$ per month; Chadee et al., 2014; Yeaw et al., 2012). Furthermore, access to testing and a blood glucose meter is often a health barrier to under-resourced communities (e.g., rural and low-income; Sherwani et al., 2016). As such, the current methods for diabetes management raise concerns regarding 
their invasive, time-consuming, and expensive approach.

Recent technological innovations have led to the development of electronic health devices that address the limitations of current standards of assessment (i.e., Apple watch and FitBit; Dinh-Le et al., 2019). These devices have improved the accessibility and convenience of health assessments (Dias \& Paulo Silva Cunha, 2018). Of particular interest is the development of TOI. Since it has been implemented into the smartphone application Anura, TOI has enabled various electronic gadgets to function as versatile health devices without increasing costs. If TOI can aid in HbA1c measurements, then it can potentially overcome the limitations of current methods by utilizing a smartphone to capture video images of the face for extraction of $\mathrm{HbA} 1 \mathrm{c}$ in a convenient, contactless, and remote manner (Wei et al., 2018).

TOl works by capitalizing on the translucent nature of facial skin and uses machine learning algorithms to obtain facial blood flow (Wei et al., 2018). Optical sensors on smartphones can capture the red light re-emitted from hemoglobin underneath the skin in order to extract facial blood flow changes (Wei et al., 2018). Various health markers can be predicted from TOI because facial blood flow changes are tightly associated with cardiovascular change (Wei et al., 2018). Since $\mathrm{HbA1c}$ levels are associated with changes in the cardiovascular system, predictive measurements of $\mathrm{HbA} 1 \mathrm{c}$ are considered an appropriate application of TOI. Moreover, a recent study established the approval concept of HbA1c optical detection and found optical methods to accurately estimate the percentage of HbA1c (Mandal \& Manasreh, 2018).

These results provide insight into the potential success of TOl-based $\mathrm{HbA} 1 \mathrm{c}$ prediction. Furthermore, the literature suggests that TOI is highly efficient and accurate in predicting various health markers. TOl has been demonstrated to be a strong predictor of heart rate and heart rate variability (Wei et al., 2018). Camerabased health readings using Anura (i.e., TOI), were found to have statistically significant correlates with Food and Drug Administration (FDA)-approved medical devices (Wei et al., 2018). In addition, by applying advanced machine learning methods, TOI has been found to effectively predict blood pressure levels (Luo et al., 2019). The computational models were found to predict systolic and diastolic blood pressure with $95 \%$ and $96 \%$ accuracy, respectively (Luo et al., 2019).

In light of this, the present study aims to expand the capabilities of TOI to measure HbA1c levels. We investigated whether a novel computational model can be used to predict HbA1c measures. To create the model, the machine learning algorithm was trained to produce random forest classifiers for diabetes prediction. Through 
the use of training sets of data, the model was provided with predictive features and labels to classify individuals as nondiabetic or diabetic. Following training, the model was evaluated using a test data set.

Model validation is of particular interest since the development of an authenticated non-invasive, convenient, and inexpensive device for HbA1c measurement would allow individuals to confidently monitor their HbA1c levels with a smartphone and mitigate diabetes-related complications. Thus, we used a kitchen sink random machine-learning algorithm to utilize the blood flow information obtained from TOI to make diabetes predictions. We then examined the validity of the computational model in comparison to the FDA-approved immunoassay for identifying critical HbA1c levels. To examine the accuracy of our model, we compared the model's predictions from blood flow extracted by TOI with collected blood samples. We hypothesized that if the model has a high classification accuracy and high ROC Area Under the Curve (AUC) score, then the findings suggest the potential use of TOI as a valid methodology for assessing HbA1c conveniently, non-invasively, inexpensively, and remotely.

\section{Methods}

\section{Participants}

513 participants (385 males; age, $M=$
42.81 years, $S D=14.33$ ) were recruited during their annual physical examination at the Health Management Centre of the Affiliated Hospital of Nanjing University Medical School, China. All participants were given full disclosure of the research protocol and signed a written informed consent form prior to the experiment. The study was conducted in accordance with the NIH research ethics guidelines and was approved by the Research Ethics Review Committee.

\section{Materials}

The transdermal optical imaging (TOI) system, the Anura smartphone application, was used to collect participants' facial blood flow information. Anura uses TOl to detect and track an individual's facial regions of interest (ROIs), which provide the optical properties of the face to obtain blood flow information. A portable screen was used to collect participant's Anura video recordings. The portable screen includes an iPhone 7 smartphone positioned on a tripod and an LED light positioned above the smartphone. The LED light emitted uniform lighting from the smartphone's camera point of view (see Figure 1).

\section{Procedures}

Participants were tested individually in an examination room at the Health Management Centre of the Affiliated Hospital of Nanjing University Medical 


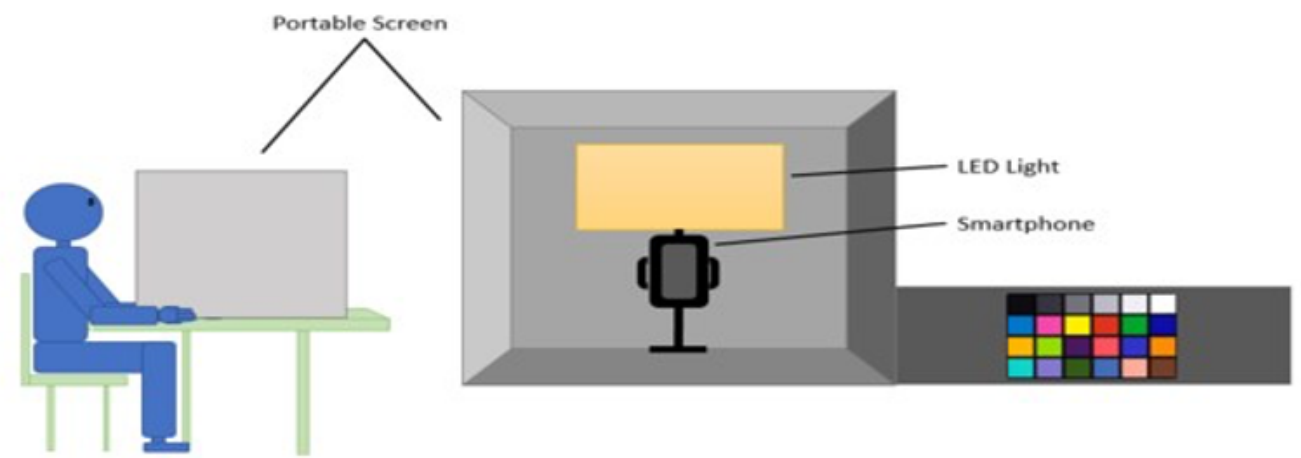

Figure 1. Glycated hemoglobin A1c (HbA1c) measurements are collected in this AnuraBP series setup.

Participants sit in front of a table, which holds a portable screen. The portable screen consists of an iPhone 7 smartphone positioned on a tripod and an LED light positioned above the smartphone.

School. First, participants' blood samples were collected by medical professionals. Next, participants were instructed to remain seated in front of a desk that held the portable screen. The Anura smartphone application recorded a 1 -minute video of the participant's face in order to measure and collect their facial blood flow information.

\section{Data Analysis}

Blood Analysis. Blood samples were collected from the participants and tested in the laboratory for glycated hemoglobin A1 measurements.

TOI Analysis. With the use of TOI analysis, the participants' hemoglobin concentration (HC) was collected from a 1-minute video from the digital camera in the smartphone (Wei et al., 2018). This analysis takes advantage of the fact that the human facial skin is translucent. When light hits our skin, it travels beneath the skin and re-emits after travelling through different skin tissues (Wei et al., 2018). The optical cameras in the smartphone capture these properties of the skin in order to obtain information on skin chromophore content. Since the dominant chromophores, hemoglobin and melanin, have different colour signatures, we can utilize the distinction to visualize hemoglobin in the dermis (Wei et al., 2018). TOI and machine learning algorithms can isolate hemoglobin-rich regions and generate corresponding images of participants' hemoglobin concentration (Wei et al., 2018).

$\mathrm{HC}$ is obtained within the video stream using bit-plane slicing to isolate a set of bit-planes that provide high a signal-tonoise ratio (SNR) with regard to facial cardiovascular activities (Wei et al., 2018). The high SNR bit-planes were determined following training sets of images that were concurrently taken with FDA-approved medical instruments that measure cardiovascular activities on the face (Wei et al., 2018). The signals from pixels 
within the bit-planes are employed to systematically produce images that contain the corresponding $\mathrm{HC}$ in each pixel (Wei et al., 2018). These images are then put together as videos to reflect $\mathrm{HC}$ changes in all parts of the face (Wei et al., 2018).

Machine Learning Analysis. The training dataset was divided into two parts: nondiabetes and diabetes. We used a novel kitchen sink random forest model to utilize the facial blood flow information obtained from TOI for diabetes classification. Since HbA1c accounts for $97 \%$ of total hemoglobin, we constructed a model to particularly extract HbA1c from TOl's obtained hemoglobin concentration (Kahn \& Fonseca, 2008). Thus, the video of each participant's face was analyzed for facial blood flow information that reflects cardiovascular activities that correlate with HbA1c.

In order to construct the Random Forest (RF) model, we used MATLAB to build notbalanced decision trees for ensemble diabetes prediction. Random samples of the training dataset were used to build the not-balanced trees. Each decision tree was built to make independent diabetes classification and 'vote' for the corresponding class (i.e., non-diabetes or diabetes) based upon HbA1c thresholds. In addition, we used MATLAB bagging and feature randomness methods to produce uncorrelated decision trees. We also applied the MATLAB feature selection and not-balanced RF classifier training with a threshold for non-diabetes and diabetes equal to clinically approved ones, $5.7 \%$ (Sherwani et al., 2016).

The results from the laboratory HbA1c test were used as ground truth data in order to evaluate the accuracy of our diabetes classification model.

Validation of the Model. To validate the model, data were derived from pristine testing on 400 pristine participants pseudo-randomly selected during 20 trials of training and testing. To compare the data obtained from TOl against those from the blood samples, we computed the MATLAB confusion matrix and ROC curve of RF classifier to assess the level of agreement between TOI and the blood samples' diabetes prediction. The confusion matrix was used to assess the number of false positives, false negatives, true positives, and true negatives of TOI. The ROC curve of RF classifier was used to assess the true positive rate and false positive rate of TOI. The threshold for diabetes was incorporated in the MATLAB feature selection and not-balanced random forest classifier training at $5.7 \%$ (see Figures $2 \& 3$ ).

\section{Results}

In the present study, pristine testing was done on 400 pristine participants pseudorandomly selected during 20 trials of training/testing. Using MATLAB, the diabetes classification model was 
evaluated by a confusion matrix and area under the ROC curve (AUC; see Figures 2 \& 3).

The confusion matrix visualizes the performance of the model in predicting diabetes class (see Figure 2).

\section{Confusion Matrix}

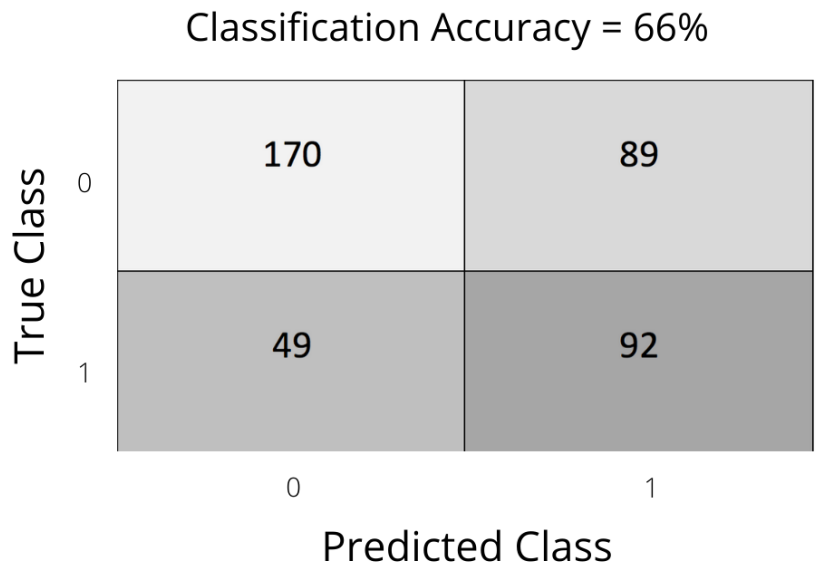

Figure 2. The confusion matrix. There are two axes: (1) the true class (y-axis; determined by blood samples), and (2) the predicted class (x-axis; determined by TOI). Non-diabetes is classified as ' 0 ', and diabetes is classified as ' 1 '. The equal error rate (EER) threshold values for the model's false positive and false negative rate is set to $\left\{\begin{array}{llll}159 & 100 & 55 & 86\end{array}\right\}$ 0.351 . TOl has a classification accuracy of $66 \%$.

The accuracy of the predicted class (determined by TOI) is evaluated by the true class (determined by blood samples). The top-left and bottom-right entry of the confusion matrix shows the predicted class's true positive (TP) and true negative predictions (NP) respectively. The topright and bottom-left entries of the confusion matrix show the predicted class's false positive (FP) and false negative (FN) predictions, respectively.
The classification accuracy of the confusion matrix is calculated as the total number of correct predictions (TP + TN) divided by the total number of the dataset $(P+N)$.

- Correct predictions:

$$
\text { - } 262 \text { predictions }
$$

- Total number of the dataset:

- 400 pristine participants

- Classification accuracy: - 66\% ( = 262/499; see Figure 2)

The ROC curve of the RF classifier is a graphical illustration of a model's prediction of binary outcomes. It was used to assess the true positive rate and false positive rate of TOI (see Figure 3).

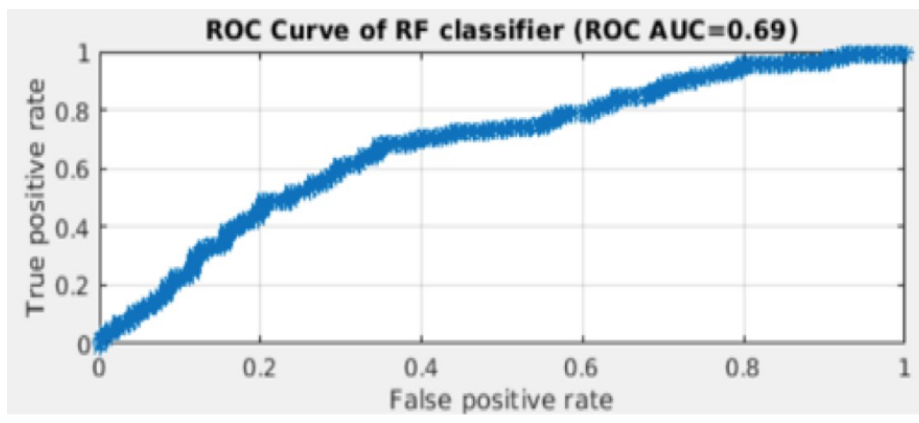

Figure 3. The ROC curve of the RF classifier. The ROC curve is generated by plotting the true positive rate $(y$-axis; $F P /(F P+T N))$ against the false positive rate $(x-$ axis; $T P /(T P+F N))$. The true and false positive rates evaluate the accuracy of the model's classifications. It is the probability in which the model correctly or incorrectly classifies the individual respectively. The TOI has a ROC AUC of 0.69 .

The ROC AUC indicates how well the model can distinguish between the two classes (i.e., non-diabetes and diabetes). Using the ROC curve, the ROC AUC can be determined as per the following: 
1. The ROC curve of the RF classified

a.Generated by plotting the true positive rate $(y$-axis; $T P /(T P+F N))$ against the false positive rate $(X-$ axis; $F P /(F P+T N))$

i. True positive rate $=92 /(92+49)$ $=0.65$

ii. False positive rate $=89 /(89+$ 170) $=0.34$

2. Calculation of the ROC AUC

a. ROC's sum(height ${ }^{*}$ weight)

3. The ROC AUC

a.0.69 (see Figure 3)

\section{Discussion}

The limitations of conventional blood glucose monitoring techniques are a pressing medical issue. In this study, systematic efforts were made in designing a random forest model for diabetes prediction. Novel machine learning classification algorithms were studied and evaluated on their performance on various measures. An AUC of .5 and a classification accuracy of $50 \%$ suggests that the classification model is unable to distinguish between the positive and negative class. So, the benchmark in which ROC AUC and classification accuracy are considered high, is above .5 and 50\%, respectively. Using blood $\mathrm{HbA1C}$ test results as ground truth data, our model was found to have a high classification accuracy and ROC AUC score, providing evidence that our model has good class separation capacity. The majority of TOl's diabetes classification predictions were found to correctly classify individuals as non-diabetic or diabetic. Thus, the results indicate promise for TOI as a potential alternative method for diabetes identification without a blood test.

While laboratory testing is the most effective in assessing $\mathrm{HbA1c}$, it requires invasive and painful extraction of blood samples and it is costly. In contrast, by using TOI technology and machine learning algorithms, diabetes prediction can be simplified and made more accessible. Nevertheless, it is important to note that TOI can only serve as a supplementary source for diabetes management; it is not a substitute for the clinical judgment of a healthcare professional.

Though the results of this study are promising, more evidence is needed before widespread application. Such application is restricted due to the considerable limitations of the current study. For example, the effectiveness of TOI may only be applicable to certain conditions and groups. The study was conducted in a highly controlled environment, participants remained seated with a smartphone on a tripod at a fixed distance from their face. In addition, facial recordings were obtained at fixed lighting and room temperature.

In practice, recordings will be subject to variable environmental conditions via colour temperatures of light and motion and positioning of head and smartphone. Future studies should be conducted in 
diverse environmental conditions to test the robustness of TOI technology. Another limitation in the present study involves the issue of racial homogenization. Although the majority of participants in the study were of East Asian descent, there was a reasonable degree of skin tone variation that did not influence the ability of TOI. Nonetheless, the study lacks racial heterogeneity, there was not a sufficient number of participants with very dark or fair skin tones. Thus, the extent to which TOl can be generalized to a wider population remains unclear.

Future research should investigate the potency of TOl in extracting $\mathrm{HC}$ among a large sample of diverse skin tones and races. While the present study shows promise in a novel TOl technology to accurately predict diabetes, a limitation of this method is that it does not provide a wide range of diabetes classifications. To monitor diabetes, it is essential that the assessment tool provides insight into prediabetes and the risk of diabetesrelated complications. However, this study aimed to investigate the potential of TOI to extract $\mathrm{HbA} 1 \mathrm{c}$ and make diabetes predictions. Future studies should make an effort to extend this initial classification model to encompass various diabetesrelated predictions.

Although further work is necessary to address the aforementioned limitations, this study is valuable in light of overcoming the challenges in diabetes health management. Namely, it provides evidence for the potential use of the TOI technology, Anura, for truly contactless, non-invasive, and convenient assessments for diabetes management. This technology has the potential to revolutionize diabetes management by predicting diabetes classifications through the use of a digital camera in a smartphone. The application of the TOl technology for monitoring diabetes would have a wide range of economic, societal, and personal benefits.

At the individual level, TOI can provide a more proactive approach to healthcare and improve several aspects of diabetes management. A smartphone would allow people to assess and monitor their $\mathrm{HbA} 1 \mathrm{c}$ levels anytime and anywhere, helping to keep their glycemic levels within a healthy range. In addition, if a smartphone can alert an individual at risk for diabetes or diabetes-related complications, they can seek medical attention early.

Given that hyperglycemia can cause significant damage to vital organs, it is essential to adopt a proactive approach to diabetes management (University of Leicester, 2016). Collectively, TOI and machine learning algorithms are promising for the advancement of diabetes management and proactive self-care. Future studies should work to expand the initial model classifications as well as evaluate the robustness of TOI in diverse populations and variable environmental conditions. 


\section{References}

Banfield, R. E., Hall, L. O., Bowyer, K. W., \& Kegelmeyer, W. P. (2007). A comparison of decision tree ensemble creation techniques. IEEE Transactions on Pattern Aanalysis and Machine Intelligence, 29(1), 173-180. https://doi.org/10.1109/tpami.2007.250609

Bird, Y., Lemstra, M., Rogers, M., \& Moraros, J. (2015). The relationship between socioeconomic status/income and prevalence of diabetes and associated conditions: A cross-sectional population-based study in Saskatchewan, Canada. International Journal for Equity in Health, 14, 93. https://doi.org/10.1186/s12939-015-0237-0

Chadee, A., Blackhouse, G., \& Goeree, R. (2014). Point-of-Care Hemoglobin A1c Testing: A Budget Impact Analysis. Ontario health technology assessment series, 14(9), 1-23. Retrieved from https://www.hqontario.ca/Evidence-to-Improve-Care/Health-TechnologyAssessment/Journal-Ontario-Health-Technology-Assessment-Series

Deshpande, A. D., Harris-Hayes, M., \& Schootman, M. (2008). Epidemiology of diabetes and diabetes-related complications.

Physical

Therapy, 88(11),

$1254-1264$. https://doi.org/10.2522/ptj.20080020

Dias, D., \& Paulo Silva Cunha, J. (2018). Wearable Health Devices-Vital Sign Monitoring, Systems and Technologies. Sensors, 18(8), 2414.

https://doi.org/10.3390/s18082414

Dinh-Le, C., Chuang, R., Chokshi, S., \& Mann, D. (2019). Wearable Health Technology and Electronic Health Record Integration: Scoping Review and Future Directions. JMIR mHealth and uHealth, 7(9), e12861. https://doi.org/10.2196/12861

Ginsberg B. H. (2009). Factors affecting blood glucose monitoring: sources of errors in measurement. Journal of Diabetes Science and Technology, 3(4), 903-913.

https://doi.org/10.1177/193229680900300438

Kahn, R., \& Fonseca, V. (2008). Translating the A1C Assay. Diabetes Care, 31(8), $1704-$ 1707. https://doi.org/10.2337/dc08-0878

Luo, H., Yang, D., Barszczyk, A., Vempala, N., Wei, J., Wu, S. J., Zheng, P. P., Fu, G., Lee, K., \& Feng, Z. P. (2019). Smartphone-Based Blood Pressure Measurement Using Transdermal Optical Imaging Technology. Circulation. Cardiovascular imaging, 12(8), e008857. https://doi.org/10.1161/CIRCIMAGING.119.008857 
Mandal, S., \& Manasreh, M. O. (2018). An In-Vitro Optical Sensor Designed to Estimate Glycated Hemoglobin Levels. Sensors, 18(4), 1084.

https://doi.org/10.3390/s18041084

Ong, W. M., Chua, S. S., \& Ng, C. J. (2014). Barriers and facilitators to self-monitoring of blood glucose in people with type 2 diabetes using insulin: a qualitative study. Patient Prefer Adherence, 8, 237-246. https://doi.org/10.2147/PPA.S57567

Qian, J., \& Scheer, F. (2016). Circadian System and Glucose Metabolism: Implications for Physiology and Disease. Trends in Endocrinology and Metabolism, 27(5), 282-293.

https://doi.org/10.1016/j.tem.2016.03.005

Saeedi, P., Petersohn, I., Salpea, P., Malanda, B., Karuranga, S., Unwin, N., Colagiuri, S., Guariguata, L., Motala, A. A., Ogurtsova, K., Shaw, J. E., Bright, D., Williams, R., \& IDF Diabetes Atlas Committee (2019). Global and regional diabetes prevalence estimates for 2019 and projections for 2030 and 2045: Results from the International Diabetes Federation Diabetes Atlas, 9th edition. Diabetes Research and Clinical Practice, 157, 107843. https://doi.org/10.1016/j.diabres.2019.107843

Solnica, B., \& Naskalski, J. W. (2007). Quality control of self-monitoring of blood glucose: why and how? Journal of Diabetes Science and Technology, 1(2), 164-168. https://doi.org/10.1177/193229680700100204

University of Leicester. (2016, January 5). High blood sugar levels could lead to heart attack complications. ScienceDaily. www.sciencedaily.com/releases/2016/01/160105101902.htm

Wei, J., Luo, H., Wu, S. J., Zheng, P. P., Fu, G., \& Lee, K. (2018). Transdermal Optical Imaging Reveal Basal Stress via Heart Rate Variability Analysis: A Novel Methodology Comparable to Electrocardiography. Frontiers in Psychology, 9, 98. https://doi.org/10.3389/fpsyg.2018.00098

Yeaw, J., Lee, W. C., Wolden, M. L., Christensen, T., \& Groleau, D. (2012). Cost of SelfMonitoring of Blood Glucose in Canada among Patients on an Insulin Regimen for Diabetes. Diabetes Therapy, 3(1), 7. https://doi.org/10.1007/s13300-012-0007-6 